\title{
On some Ramsey and Turán-type numbers for paths and cycles
}

\author{
Tomasz Dzido \\ Institute of Mathematics, University of Gdańsk \\ Wita Stwosza 57, 80-952 Gdańsk, Poland \\ tdz@math.univ.gda.pl \\ Marek Kubale
}

Algorithms and System Modelling Department, Gdańsk University of Technology

G. Narutowicza 11/12, 80-952 Gdańsk, Poland

kubale@eti.pg.gda.pl

Konrad Piwakowski

Algorithms and System Modelling Department, Gdańsk University of Technology

G. Narutowicza 11/12, 80-952 Gdańsk, Poland

coni@eti.pg.gda.pl

Submitted: Nov 15, 2005; Accepted: Jul 3, 2006; Published: Jul 11, 2006

Mathematics Subject Classifications: 05C55, 05C15, 05C38

\begin{abstract}
For given graphs $G_{1}, G_{2}, \ldots, G_{k}$, where $k \geq 2$, the multicolor Ramsey number $R\left(G_{1}, G_{2}, \ldots, G_{k}\right)$ is the smallest integer $n$ such that if we arbitrarily color the edges of the complete graph on $n$ vertices with $k$ colors, there is always a monochromatic copy of $G_{i}$ colored with $i$, for some $1 \leq i \leq k$. Let $P_{k}$ (resp. $C_{k}$ ) be the path (resp. cycle) on $k$ vertices. In the paper we show that $R\left(P_{3}, C_{k}, C_{k}\right)=R\left(C_{k}, C_{k}\right)=$ $2 k-1$ for odd $k$. In addition, we provide the exact values for Ramsey numbers $R\left(P_{4}, P_{4}, C_{k}\right)=k+2$ and $R\left(P_{3}, P_{5}, C_{k}\right)=k+1$.
\end{abstract}

\section{Introduction}

In this paper all graphs considered are undirected, finite and contain neither loops nor multiple edges. Let $G$ be such a graph. The vertex set of $G$ is denoted by $V(G)$, the edge set of $G$ by $E(G)$, and the number of edges in $G$ by $e(G) . C_{m}$ denotes the cycle of length $m$ and $P_{m}$ - the path on $m$ vertices. For given graphs $G_{1}, G_{2}, \ldots, G_{k}, k \geq 2$, the multicolor Ramsey number $R\left(G_{1}, G_{2}, \ldots, G_{k}\right)$ is the smallest integer $n$ such that if we arbitrarily color the edges of the complete graph of order $n$ with $k$ colors, then it always contains a monochromatic copy of $G_{i}$ colored with $i$, for some $1 \leq i \leq k$. We only 
consider 3-color Ramsey numbers $R\left(G_{1}, G_{2}, G_{3}\right)$ (in other words we color the edges of $K_{n}$ with colors red, blue and green). The Turán number $T(n, G)$ is the maximum number of edges in any $n$-vertex graph which does not contain a subgraph isomorphic to $G$. By $T^{\prime}(n, G)$ we denote the maximum number of edges in any $n$-vertex non-bipartite graph which does not contain a subgraph isomorphic to $G$. A non-bipartite graph on $n$ vertices is said to be extremal with respect to $G$ if it does not contain a subgraph isomorphic to $G$ and has exactly $T^{\prime}(n, G)$ edges. By $T^{*}(n, G)$ we denote the maximum number of edges in any $n$-vertex bipartite graph which does not contain a subgraph isomorphic to $G$. For any $v \in V(G)$, by $r(v), b(v)$ and $g(v)$ we denote the number of red, blue and green edges incident to $v$, respectively. The degree of vertex $v$ will be denoted by $d(v)$ and the minimum degree of a vertex of $G$ by $\delta(G)$. The open neighbourhood of vertex $v$ is $N(v)=\{u \in V(G) \mid\{u, v\} \in E(G)\} . G_{1} \cup G_{2}$ denotes the graph which consists of two disconnected subgraphs $G_{1}$ and $G_{2} . k G$ stands for the graph consisting of $k$ disconnected subgraphs $G$. We will use $G_{1}+G_{2}$ to denote the join of $G_{1}$ and $G_{2}$, defined as $G_{1} \cup G_{2}$ together with all edges between $G_{1}$ and $G_{2}$.

The remainder of this paper is organized as follows. Section 2 contains some facts on the numbers $T^{\prime}(n, G)$, where $G$ is a cycle. We first establish the exact value of $T^{\prime}\left(n, C_{k}\right)$, where $k \leq n \leq 2 k-2$. Next, we continue in this fashion to obtain an upper bound for $T^{\prime}\left(2 k-1, C_{k}\right)$. Section 3 contains our main result that $R\left(P_{3}, C_{k}, C_{k}\right)=R\left(C_{k}, C_{k}\right)=2 k-1$, where $C_{k}$ is the odd cycle on $k$ vertices. The last Section 4 presents two new formulas for the following Ramsey numbers: $R\left(P_{4}, P_{4}, C_{k}\right)=k+2$ and $R\left(P_{3}, P_{5}, C_{k}\right)=k+1$.

\section{Values of $T^{\prime}\left(n, C_{k}\right)$}

First, we present some facts which are often used in the paper.

Definition 1 The circumference $c(G)$ of a graph $G$ is the length of its longest cycle.

Definition 2 The girth of a graph $G$ is the length of its shortest cycle.

Definition 3 A graph is called weakly pancyclic if it contains cycles of every length between the girth and the circumference.

Theorem 4 (Brandt, [3]) A non-bipartite graph $G$ of order $n$ and more than $\frac{(n-1)^{2}}{4}+1$ edges contains all cycles of length between 3 and the length of the longest cycle (thus such a graph is weakly pancyclic of girth 3).

Theorem 5 (Brandt, [4]) Every non-bipartite graph $G$ of order $n$ with minimum degree $\delta(G) \geq(n+2) / 3$ is weakly pancyclic of girth 3 or 4 .

The following notation and terminology comes from [6].

For positive integers $a$ and $b$ define $r(a, b)$ as

$$
r(a, b)=a-b\left\lfloor\frac{a}{b}\right\rfloor=a \bmod b .
$$


For integers $n \geq k \geq 3$, define $w(n, k)$ as

$$
w(n, k)=\frac{1}{2}(n-1) k-\frac{1}{2} r(k-r-1),
$$

where $r=r(n-1, k-1)$.

Woodall's theorem [12] can then be written as follows.

Theorem 6 ([6]) Let $G$ be a graph on $n$ vertices and $m$ edges with $m \geq n$ and $c(G)=k$. Then

$$
m \leq w(n, k)
$$

and this result is the best possible.

First, we state the following lemma.

Lemma 7 If $n \geq 2 k-3$ and $k \geq 1$, then $T^{*}\left(k K_{2}, n\right)=(k-1) n-(k-1)^{2}$.

Proof. The proof is by induction on $k$. It is clear that $T^{*}\left(K_{2}, n\right)=0$ for any integer $n$. It is easy to see that $K_{1, r}$ for $r \geq 1$ and $K_{3}$ are the only connected graphs which do not contain $K_{2} \cup K_{2}$. Thus we obtain $T^{*}\left(2 K_{2}, n\right)=n-1$ for all $n$, since $K_{3}$ is not bipartite.

Let $G$ be any nonempty bipartite graph of order $n$, which does not contain $k K_{2}$. Choose any edge $v w$. Define $H$ to be the subgraph induced by $V(G)-\{v, w\}$. Obviously $H$ cannot contain $(k-1) K_{2}$, so by the induction hypothesis $e(H) \leq(k-2)(n-2)-(k-2)^{2}$. Since $G$ is bipartite, so the number of edges with at least one vertex in $\{v, w\}$ is not greater than $n-1$. Thus we obtain $e(G) \leq(k-2)(n-2)-(k-2)^{2}+(n-1)=(k-1) n-(k-1)^{2}$, which implies $T^{*}\left(k K_{2}, n\right) \leq(k-1) n-(k-1)^{2}$. The graph $K_{k-1, n-k+1}$ implies that $T^{*}\left(k K_{2}, n\right) \geq(k-1) n-(k-1)^{2}=(k-1)(n-k+1)$.

Lemma 8 Let $G$ be a bipartite graph of order $2 k-2$ with $k^{2}-3 k+4$ edges, where $k$ is odd and $k \geq 9$. Then any two vertices, which belong to different sides of the bipartition, are joined by a path of length $k-2$.

Proof. Let $\{X, Y\}$ be the bipartition of $G$ and choose any two vertices $x \in X, y \in Y$. Graph $G$ can be seen as a complete bipartite graph without at most $k-3$ edges. Define $X^{\prime}=(X \backslash\{x\}) \cap N(y)$ and $Y^{\prime}=(Y \backslash\{y\}) \cap N(x)$. The number of edges in $G$ guarantees that $\left|X^{\prime}\right| \geq 1,\left|Y^{\prime}\right| \geq 1$ and $\left|X^{\prime}\right|+\left|Y^{\prime}\right| \geq 2 k-4-(k-3)=k-1$. Thus the complete bipartite graph with bipartition $\left\{X^{\prime}, Y^{\prime}\right\}$ contains at least $k-2$ edges, so at least one of them, say $v w$, where $v \in X^{\prime}$ and $w \in Y^{\prime}$ must belong to $G$ as well. In this way we obtain path $x w v y$, which is a path of length 3 joining $x$ and $y$. Now we will show that any path of length at least 3 but shorter than $k-2$ which joins $x$ and $y$ can be extended by two additional vertices to a longer path joining $x$ and $y$, which by induction completes the proof.

Assume that $x$ and $y$ are joined by a path $P$ of length $k-p$, where $4 \leq p \leq k-3$. Define $G^{\prime}=G[V(G) \backslash V(P)]$. We have $e\left(G^{\prime}\right)=e(G)-e(P)-\mid\{v w \in E(G): v \in P, w \in$ 
$\left.G^{\prime}\right\} \mid \geq k^{2}-3 k+4-(k-p+1)^{2} / 4-(k-p+1)(k+p-3) / 2$. From Lemma 7 we have $T^{*}\left((p / 2+1) K_{2}, k+p-3\right)=\left(p^{2}+2 k p-6 p\right) / 4$. One can easily verify that this implies $e\left(G^{\prime}\right) \geq T^{*}\left((p / 2+1) K_{2}, k+p-3\right)$ and thus $G^{\prime}$ contains $p / 2+1$ independent edges. Assume that there is no path of order $k-p+2$ joining $x$ and $y$ in graph $G$. In this case any edge from $G^{\prime}$ can be connected to at most $(k-p+1) / 2$ vertices from $P$ or in other words cannot be connected to at least $(k-p+1) / 2$ vertices from $P$. So we have $e(G) \leq e\left(K_{k-1, k-1}\right)-\left|\left\{v w \notin E(G): v \in P, w \in G^{\prime}\right\}\right| \leq(k-1)^{2}-(p / 2+1)(k-p+1) / 2=$ $k^{2}-(10+p) k / 4+\left(p^{2}+p+2\right) / 4<k^{2}-3 k+4=e(G)$, a contradiction. Hence there must be a path of order $k-p+2$ joining $x$ and $y$ in graph $G$.

Theorem 9 For odd integers $k \geq 5$

$$
T^{\prime}\left(n, C_{k}\right)=w(n, k-1),
$$

where $k \leq n \leq 2 k-2$.

Proof. The last part of the thesis of Theorem 6 implies that $T^{\prime}\left(n, C_{k}\right) \geq w(n, k-1)$. Let us suppose that there exists a non-bipartite $C_{k}$-free graph $G^{\prime}$ on $n$ vertices which has more than $w(n, k-1)$ edges. Observe that $w(n, k)$ is not a decreasing function of $k$ and of $n$, i.e. $w\left(n, k_{1}\right) \geq w\left(n, k_{2}\right)$ if $k_{1}>k_{2}$ and $w\left(n_{1}, k\right) \geq w\left(n_{2}, k\right)$ if $n_{1}>n_{2}$. Then, graph $G^{\prime}$ must contain a cycle of length greater than $k$. Now, we prove that $w(n, k-1)+1>\frac{(n-1)^{2}}{4}+1$. The maximal possible value of $n$ is $2 k-2$. Then, the left-hand side is equal to $k^{2}-3 k+4$ and the right-hand side is equal to $k^{2}-3 k+\frac{13}{4}$, so by Brandt's theorem graph $G^{\prime}$ contains $C_{k}$. For the case $n=2 k-3$ we obtain that $r(n-1, k-2)=0$ and $w(n, k-1)+1>\frac{(n-1)^{2}}{4}+1$, and $G^{\prime}$ also contains a cycle of length $k$. For the case $n \leq 2 k-4$ we have that $r(n-1, k-2)=n-(k-1)$. Then, $w(n, k-1)+1=\frac{1}{2} n^{2}+k^{2}-k n-3 k+\frac{3}{2} n+3$ and the inequality $w(n, k-1)+1>\frac{(n-1)^{2}}{4}+1$ implies the following inequality: $\frac{n^{2}}{4}+n(2-k)+k^{2}+\frac{7}{4}>3 k$. The minimal value of the left-hand side holds for $n=2 k-4$ and it is equal to $4 k-2.25$, so for $k \geq 3$ graph $G^{\prime}$ contains a cycle of length $k$.

Theorem 10 For odd integers $k \geq 9$

$$
T^{\prime}\left(2 k-1, C_{k}\right) \leq \frac{(2 k-2)^{2}}{4}-1=(k-1)^{2}-1 .
$$

Proof. Let $G$ be a non-bipartite graph of order $2 k-1$. By Theorem 4 and by property $w(2 k-1, k-1)=k^{2}-3 k+5<\frac{(2 k-2)^{2}}{4}+2$ we obtain that if $G$ has at least $\frac{(2 k-2)^{2}}{4}+2$ edges, then it contains $C_{k}$.

Assume that $G$ has $\frac{(2 k-2)^{2}}{4}+1=k^{2}-2 k+2$ edges. Suppose that there is a vertex $v \in V(G)$ such that $d(v) \leq k-2$. If $G-v$ is a non-bipartite subgraph, we immediately 
obtain a contradiction with $T^{\prime}\left(2 k-2, C_{k}\right)=k^{2}-3 k+3$, so $G-v$ must be bipartite. It is clear that vertex $v$ must be joined to at least one vertex in each side of the bipartition of $G-v$. Applying Lemma 8 we find a cycle $C_{k}$ in graph $G$, so we have that $\delta(G)=k-1$. In this case, the number of edges of graph $G$ is at least $\frac{(2 k-1)(k-1)}{2}=k^{2}-\frac{3}{2} k+\frac{1}{2}>k^{2}-2 k+2$, a contradiction. These observations lead us to the conclusion that a non-bipartite graph $G$ on $2 k-1$ vertices and $\frac{(2 k-2)^{2}}{4}+1$ edges must contain a cycle $C_{k}$.

Consider the last case when $G$ has $(k-1)^{2}$ edges. Since $w(2 k-1, k-1)<(k-1)^{2}$ for $k>4$ and $w(k, n)$ is a non-decreasing function of $k$ and $n$, graph $G$ must contain a cycle of length at least $k$. It follows that $\delta(G) \geq k-2$. We obtain this property using the same arguments as those in the previous case. Since $k-2 \geq(2 k+1) / 3$ for $k \geq 7$, then by Theorem 5 graph $G$ is weakly pancyclic of girth 3 or 4 , so it contains a cycle of length $k$.

Finally, for the sake of completeness we recall a few Turán numbers for short paths. In 1975 Faudree and Schelp proved

Theorem 11 ([9]) If $G$ is a graph with $|V(G)|=k t+r, 0 \leq r<k$, containing no path on $k+1$ vertices, then $|E(G)| \leq t\left(\begin{array}{l}k \\ 2\end{array}\right)+\left(\begin{array}{l}r \\ 2\end{array}\right)$ with equality if and only if $G$ is either $\left(t K_{k}\right) \cup K_{r}$ or $\left((t-l-1) K_{k}\right) \cup\left(K_{(k-1) / 2}+\bar{K}_{(k+1) / 2+i k+r}\right)$ for some $l, 0 \leq l<t$, when $k$ is odd, $t>0$, and $r=(k \pm 1) / 2$.

It is easy to check that we obtain the following

Corollary 12 For all integers $n \geq 3$

$$
\begin{gathered}
T\left(n, P_{3}\right)=\left\lfloor\frac{n}{2}\right\rfloor \\
T\left(n, P_{4}\right)= \begin{cases}n & \text { if } n \equiv 0 \quad \bmod 3 \\
n-1 & \text { otherwise. }\end{cases} \\
T\left(n, P_{5}\right)= \begin{cases}\frac{3 n}{2} & \text { if } n \equiv 0 \quad \bmod 4 \\
\frac{3 n}{2}-2 & \text { if } n \equiv 2 \quad \bmod 4 \\
\frac{3 n}{2}-\frac{3}{2} & \text { otherwise }\end{cases}
\end{gathered}
$$

\section{Ramsey numbers for odd cycles}

In 1973 Bondy and Erdős proved that

Theorem 13 ([2]) For odd integers $k \geq 5$

$$
R\left(C_{k}, C_{k}\right)=2 k-1
$$


In 1983 Burr and Erdős gave the following Ramsey number.

Theorem $14([5])$

$$
R\left(P_{3}, C_{3}, C_{3}\right)=11
$$

In 2005 the first author determined two further numbers of this type.

Theorem $15([8])$

$$
\begin{gathered}
R\left(P_{3}, C_{5}, C_{5}\right)=9 \\
R\left(P_{3}, C_{7}, C_{7}\right)=13
\end{gathered}
$$

Now, we prove our the main result of the paper.

Theorem 16 For odd integers $k \geq 9$

$$
R\left(P_{3}, C_{k}, C_{k}\right)=R\left(C_{k}, C_{k}\right)=2 k-1
$$

Proof. Let the complete graph $G$ on $2 k-2$ vertices be colored with two colors, say blue and green, as follows: the vertex set $V(G)$ of $G$ is the disjoint union of subsets $G_{1}$ and $G_{2}$, each of order $k-1$ and completely colored blue. All edges between $G_{1}$ and $G_{2}$ are colored green. This coloring contains neither monochromatic (blue or green) cycle $C_{k}$ nor a monochromatic (red) path of length 2 . We conclude that $R\left(P_{3}, C_{k}, C_{k}\right) \geq 2 k-1$.

Assume that the complete graph $K_{2 k-1}$ is 3 -colored with colors red, blue and green. By Corollary 12, in order to avoid a red $P_{3}$, there must be at most $k-1$ red edges. Suppose that $K_{2 k-1}$ contains at most $k-1$ red edges and contains neither a blue nor a green $C_{k}$. Since the number of blue and green edges is greater or equal to $\left(\begin{array}{c}2 k-1 \\ 2\end{array}\right)-(k-1)=2(k-1)^{2}$, at least one of the blue or green color classes (say blue) contains at least $(k-1)^{2}$ edges. If the blue color class is bipartite, then one of the partition sets has at least $k$ vertices. Since $R\left(P_{3}, C_{k}\right)=k$ for $k \geq 5$ [11], the graph induced by this partition has to contain a red $P_{3}$ or a green $C_{k}$, so blue edges enforce a non-bipartite subgraph of order $2 k-1$ with at least $(k-1)^{2}$ edges which by Theorem 10 contains a blue $C_{k}$.

\section{The Ramsey numbers $R\left(P_{l}, P_{m}, C_{k}\right)$}

This section makes some observations on 3-color Ramsey numbers for two short paths and one cycle of arbitrary length.

In [1] we find two values of Ramsey numbers: $R\left(P_{4}, P_{4}, C_{3}\right)=9$ and $R\left(P_{4}, P_{4}, C_{4}\right)=7$. By using simple combinatorial properties (without the aid of computer calculations) we proved: $R\left(P_{4}, P_{4}, C_{5}\right)=9$ and $R\left(P_{4}, P_{4}, C_{6}\right)=8$ (see [7] for details).

\section{Theorem 17}

$$
R\left(P_{4}, P_{4}, C_{7}\right)=9
$$


Proof. The proof of $R\left(P_{4}, P_{4}, C_{7}\right) \geq 9$ is very simple, so it is left to the reader. Let the vertices of $K_{9}$ be labeled $1,2, \ldots, 9$. Since $R\left(P_{4}, P_{4}, C_{6}\right)=8$, we can assume $1,2,3,4,5,6$ to be the vertices of green $C_{6}$. If the subgraph induced by green edges of $K_{9}$ is bipartite, then since $R\left(P_{4}, P_{4}\right)=5$, we immediately obtain a red or a blue $P_{4}$. Since $T\left(9, P_{4}\right)=9$, the number of green edges is at least $18>\frac{(9-1)^{2}}{4}+1$, so the non-bipartite subgraph induced by green edges of $K_{9}$ is weakly pancyclic. Since $R\left(P_{4}, P_{4}, C_{3}\right)=9$, this subgraph contains green cycles of every length between 3 and the green circumference. Avoiding a green cycle $C_{7}$ we know that the number of green edges from vertices 7,8,9 to the green cycle is at most 3 . We have to consider the two following cases.

1. There is a vertex $v \in\{7,8,9\}$ which has three green edges to the vertices of green cycle $C_{6}$. We can assume that the edges $\{1,7\},\{3,7\},\{5,7\}$ are green. In this case the edges $\{2,4\},\{4,6\},\{2,6\}$ are red or blue. Without loss of generality we can assume that $\{2,4\}$ and $\{4,6\}$ are red. This enforces $\{2,7\},\{6,7\}$ to be blue and $\{2,8\},\{6,8\}$ to be green, and we obtain a green cycle of length 8 and then a green $C_{7}$.

2. There is a vertex $v \in\{7,8,9\}$ which has two green edges to the vertices of green cycle $C_{6}$. We have to consider two subcases.

(i) The edges $\{1,7\},\{3,7\}$ are green and $\{2,7\},\{4,7\},\{5,7\},\{6,7\}$ are red or blue. This enforces $\{2,6\}$ and $\{2,4\}$ to be red or blue. We obtain two situations. In the first, if edge $\{2,6\}$ is red and $\{2,4\}$ blue, then we can assume that edge $\{2,7\}$ is blue, then $\{5,7\}$ is red and we obtain a red or a blue $P_{4}$ with edge $\{6,7\}$. In the second, if edges $\{2,6\}$ and $\{2,4\}$ are red, then $\{4,7\},\{6,7\}$ are blue and $\{4,8\},\{6,8\},\{4,9\},\{6,9\}$ are green. Edge $\{2,6\}$ cannot be green. If edge $\{5,8\}$ is red, then we obtain a blue $P_{4}: 2-5-7-6$ and if $\{5,8\}$ is blue, then we have a red $P_{4}: 6-2-5-7$.

(ii) The edges $\{1,7\},\{4,7\}$ are green and $\{2,7\},\{3,7\},\{5,7\},\{6,7\}$ are red or blue. Then vertex 8 and vertex 9 have green edges to at most one vertex from $\{2,3,5,6\}$, otherwise we have either the situation considered in (i) or a green cycle of length 8. By simple considering red and blue edges from $\{7,8,9\}$ to $\{2,3,5,6\}$, we obtain a red or a blue $P_{4}$.

We obtain that there are at least 15 non-green edges from $\{7,8,9\}$ to the vertices of the green $C_{6}$. We can assume that there are at least 8 blue edges among them and we immediately have a blue $P_{4}$.

Theorem 18 For all integers $k \geq 6$

$$
R\left(P_{4}, P_{4}, C_{k}\right)=k+2 .
$$


Proof. The critical coloring which gives us the lower bound $k+2$ is easy to obtain, so we only give a proof for the upper bound. This proof can be easily deduced from Turán numbers and the theorems given above. By Theorem 9 and Corollary 12 we obtain that $T^{\prime}\left(k+2, C_{k}\right)=\frac{1}{2} k^{2}-\frac{3}{2} k+7$ for $k \geq 5$ and $T\left(k+2, P_{4}\right) \leq k+2$. It is easy to check that $T^{\prime}\left(k+2, C_{k}\right)$ is greater than the maximal number of edges in a bipartite graph on $k+2$ vertices, thus $T\left(k+2, C_{k}\right)=T^{\prime}\left(k+2, C_{k}\right)$. Suppose that we have a 3 -coloring of the complete graph $K_{k+2}$. This graph has $\frac{1}{2} k^{2}+\frac{3}{2} k+1$ edges. Note that $T\left(k+2, C_{k}\right)+2 T\left(k+2, P_{4}\right) \leq \frac{1}{2} k^{2}+\frac{1}{2} k+11<\frac{1}{2} k^{2}+\frac{3}{2} k+1$ for all $k>10$. If $k \in\{8,9,10\}$, we obtain that $T\left(k+2, C_{k}\right)+2 T\left(k+2, P_{4}\right) \leq\left(\begin{array}{c}k+2 \\ 2\end{array}\right)$ with equality for $k=8$ and $k=10$, so $R\left(P_{4}, P_{4}, C_{9}\right)=11$. By Theorem 11 we know the properties of the extremal graphs with respect to $P_{4}$ and by Theorem 9 and [6] we can describe the extremal graphs with respect to $C_{k}$, so it is easy to check that the theorem holds for the remaining cases when $k \in\{8,10\}$.

The following lemma will be useful in further considerations.

Lemma 19 Suppose that graph $G$ has $k+1$ vertices and it contains a cycle $C_{k}$ and suppose that we have a vertex $v \notin V\left(C_{k}\right)$, which is joined by $r$ edges to $C_{k}$, where $2 \leq r \leq k$. Then one of the following two possibilities holds:

(i) $G$ contains a cycle $C_{k+1}$.

(ii) $G^{\prime}=G\left[V\left(C_{k}\right)\right]$ contains at most $\frac{k(k-1)}{2}-\frac{r(r-1)}{2}$ edges.

Proof. Let $C=x_{1} x_{2} x_{3} \ldots x_{k}$ be a cycle $C_{k}$ and $v \notin V(C)$ be a vertex, which is joined by $d(v)=r$ edges to $C$, where $2 \leq r \leq k$. First, if $r \geq\left\lceil\frac{k}{2}\right\rceil$, then we immediately have a cycle $C_{k+1}$ and $(i)$ follows. Assume that $2 \leq r \leq\left\lceil\frac{k}{2}-1\right\rceil$. Let the vertices of $C$, which are joined by an edge to vertex $v$, be labeled $p_{i_{1}}, p_{i_{2}}, \ldots, p_{i_{r}}$. If any two of them are adjacent, then we obtain the cycle $C_{k+1}$ and $(i)$ follows. Otherwise, consider the following vertices: $p_{i_{1}+1}, p_{i_{2}+1}, \ldots, p_{i_{r}+1}$. In order to avoid a cycle $C_{k+1}$, these vertices must be mutually nonadjacent and $G^{\prime}$ contains at most $\frac{k(k-1)}{2}-\frac{r(r-1)}{2}$ edges.

Theorem 20 For all integers $k \geq 8$

$$
R\left(P_{3}, P_{5}, C_{k}\right)=k+1 \text {. }
$$

Proof. A critical coloring which gives us the lower bound $k+1$ is very simple, so all we need is the upper bound. It is easy to see that simply using Turán numbers does not give us the proof. Indeed, the sum $T\left(k+1, P_{3}\right)+T\left(k+1, P_{5}\right)+T\left(k+1, C_{n}\right)$ is far greater than the maximal number of edges in the complete graph on $k+1$ vertices. Suppose that we have a 3-coloring of $K_{k+1}$ with colors red, blue and green which neither contains a red $P_{3}$, nor a blue $P_{5}$, nor a green $C_{k}$. $K_{k+1}$ has to contain a green cycle $C_{k-1}$. Indeed, suppose 
that this is not the case. Since $T\left(k+1, P_{3}\right)+T\left(k+1, P_{5}\right)+T\left(k+1, C_{k-1}\right)<\left(\begin{array}{c}k+1 \\ 2\end{array}\right)$ for $k>11$, we obtain either a red $P_{3}$ or a blue $P_{5}$. For the case of $k \in\{8,9,10,11\}$ we use the properties of the extremal graphs with respect to $P_{3}$ and $P_{5}$ and we also obtain either a red $P_{3}$ or a blue $P_{5}$. Let the vertices of $K_{k+1}$ be labeled $v_{0}, v_{1}, \ldots, v_{k}$. We can assume the first $k-1$ vertices to be the vertices of green $C_{k-1}$. It is easy to see that $b\left(v_{k-1}\right)$ and $b\left(v_{k}\right)$ are greater or equal to $k-\lfloor(k-1) / 2\rfloor-1$. Note that in order to avoid a blue $P_{5}$ we obtain that the vertices $v_{k-1}$ and $v_{k}$ have no common vertex which belongs to $V\left(C_{k-1}\right)$ and which is joined by a blue edge to them. If the vertex $v_{k-1}$ or $v_{k}$ is joined by at least 4 green edges to the vertices of $C_{k-1}$, then by Lemma 19 and $R\left(P_{3}, P_{5}\right)=5$ we have a blue $P_{5}$. If $v_{k-1}$ and $v_{k}$ are joined by at most 3 green edges to the vertices of $C_{k-1}$, then by Lemma 19 and $R\left(P_{3}, P_{4}\right)=4$ we obtain a blue $P_{4}$. If $k \geq 9$ then we also have a blue $P_{5}$. In the case $k=8$ by simple considering possible colorings of the edges of $v_{k-1}$ and $v_{k}$ we obtain either a red $P_{3}$, or a blue $P_{5}$, or else a green $C_{k}$.

\section{References}

[1] Arste J., Klamroth K., Mengersen I.: Three color Ramsey numbers for small graphs, Util. Math. 49 (1996) 85-96.

[2] Bondy J.A., Erdős P.: Ramsey numbers for cycles in graphs, J. Combin. Theory Ser. $B 14$ (1973) 46-54.

[3] Brandt S.: A sufficient condition for all short cycles, Disc. Appl. Math. 79 (1997) $63-66$

[4] Brandt S.: Sufficient conditions for graphs to contain all subgraphs of a given type, Ph.D. Thesis, Freie Universität Berlin, 1994.

[5] Burr A., Erdős P.: Generalizations of a Ramsey-theoretic result of Chvatal, J. Graph Theory 7 (1983) 39-51.

[6] Caccetta L., Vijayan K.: Maximal cycles in graphs, Disc. Math. 98 (1991) 1-7.

[7] Dzido T.: Computer experience from calculating some 3-color Ramsey numbers, Technical Report of Gdańsk University of Technology, ETI Faculty 18/03 (2003).

[8] Dzido T.: Multicolor Ramsey numbers for paths and cycles, Discuss. Math. Graph Theory 25 (2005) 57-65.

[9] Faudree R.J., Schelp R.H.: Path Ramsey numbers in multicolorings, J. Combin. Theory Ser. B 19 (1975) 150-160.

[10] Greenwood R.E., Gleason A.M.: Combinatorial relations and chromatic graphs, Canad. J. Math. 7 (1955) 1-7.

[11] Radziszowski S.P.: Small Ramsey numbers, Electronic Journal of Combinatorics, Dynamic Survey 1, revision \#10, July 2004, http://www.combinatorics.org.

[12] Woodall D.R.: Maximal circuits of graphs I, Acta Math. Acad. Sci. Hungar. 28 (1976) $77-80$. 